\title{
Attosecond x-ray scattering from a particle-hole wave packet
}

\author{
M. Grosser, ${ }^{1}$ J. M. Slowik, ${ }^{1,2,3}$ and R. Santra ${ }^{1,2,3, *}$ \\ ${ }^{1}$ Department of Physics, University of Hamburg, Jungiusstrasse 9, 20355 Hamburg, Germany \\ ${ }^{2}$ Center for Free-Electron Laser Science, DESY, Notkestrasse 85, 22607 Hamburg, Germany \\ ${ }^{3}$ The Hamburg Centre for Ultrafast Imaging, Luruper Chaussee 149, 22761 Hamburg, Germany
}

(Received 1 March 2017; published 9 June 2017)

\begin{abstract}
We investigate the imaging of electron wave-packet dynamics by measuring the pattern of inelastically scattered photons. For this purpose, we develop a theory of time-resolved Compton scattering. We demonstrate that, by using a sufficiently short $\mathrm{x}$-ray pulse, the scattering cross section directly reflects the instantaneous momentum density of the electron. Therefore, we propose time-resolved Compton scattering as a tool to image electron wave-packet dynamics in momentum space. To illustrate this, we simulate electron wave packets in argon by using the time-dependent configuration-interaction singles method. Specifically, we consider coherent particle-hole wave packets, where the hole is in either the $3 p$ or the $3 s$ shell, and the particle (the excited electron) is either in a Rydberg state or in the continuum. Our calculations confirm that the dynamics of electron wave packets can indeed be imaged by measuring the doubly differential Compton scattering cross section. When the x-ray detector has no energy resolution at all, the contribution of Compton scattering to the differential scattering cross section becomes stationary. In that case, the motion of the particle and the hole can no longer be inferred from the scattering pattern.
\end{abstract}

DOI: 10.1103/PhysRevA.95.062107

\section{INTRODUCTION}

Scattering particles off a sample has long been used as a tool to probe the structure and dynamics of matter. Common choices of particles are neutrons, electrons, or photons, each having certain advantages and disadvantages [1]. The number of applications for these techniques is large. In particle physics, one commonly uses the scattering of subatomic particles in order to learn about elementary particles and their interactions [2]. At the other end of the spectrum, neutron scattering can be used to learn about the properties of solids (e.g., magnetic properties) [3,4], and x-ray scattering allows us to learn about the electronic structure of matter $[5,6]$.

Recently, the range of possible applications has increased even more due to the advent of x-ray free-electron lasers (XFELs) [7-9] and the advances in the generation of ultrashort light pulses [10,11]. Schemes have been developed that allow the generation of $x$-ray pulses with a duration on the order of 100 as [12-18]. This gives rise to the possibility of probing the dynamics of electrons on their natural timescale. In the recent past, there has been a great activity in the field of attosecond physics. A summary and commentary on those activities can be found in Refs. [11,19]. A notable success of the technique of attosecond streaking is the measurement of the delays of electrons as they are ejected from atoms in the gas phase [20-22], and from solid-state surfaces [23,24]. Another powerful technique is attosecond transient absorption spectroscopy, which allowed a real-time observation of valence-electron wave-packet dynamics [25]. High-harmonic generation [26] was used to image the hole dynamics in a molecule for the time between ionization and recombination of an electron [27]. Finally, charge migration and related electron wave-packet dynamics are interesting phenomena that

\footnotetext{
*robin.santra@cfel.de
}

are currently being investigated in the context of attosecond physics $[28,29]$.

To image electron dynamics, time-resolved x-ray scattering has been proposed [30,31]. In Ref. [31], it was assumed that the scattering pattern is determined solely by the instantaneous electron density $\rho(\mathbf{x}, t)$, according to

$$
\frac{d \sigma}{d \Omega}=\frac{d \sigma_{\mathrm{th}}}{d \Omega}\left|\int \rho(\mathbf{x}, t) e^{i \mathbf{Q} \cdot \mathbf{x}} d^{3} x\right|^{2} .
$$

Here, $d \sigma_{\mathrm{th}} / d \Omega$ is the Thomson cross section for the scattering of light by a free electron, and $\mathbf{Q}=\mathbf{k}_{\text {in }}-\mathbf{k}_{s}$ is the photon momentum transfer. This result is a straightforward generalization of the cross section for scattering from a stationary sample. It can be obtained from a semiclassical treatment of the scattering process if one assumes that the $\mathrm{x}$-ray pulse is short enough to "freeze" the dynamics of the electrons [30]. As a matter of fact, it was found that the situation is more involved in the case of time-resolved scattering (see also Refs. [30,32,33]). A rigorous description of the scattering process, within the framework of quantum electrodynamics (QED), shows that Eq. (1) is not valid anymore [30]. Instead, the scattering pattern is determined by complicated spatiotemporal correlations. This can be motivated by the fact that an ultrashort pulse has an unavoidable bandwidth, according to the energy-time uncertainty relation. In consequence, it is impossible to distinguish coherent-scattering contributions from the inelastic-scattering contributions. The differences between the semiclassical and the QED description have been illustrated by Dixit et al. for a coherent superposition of hydrogen orbitals [30]. The same has been done by Shao et al. for the case of electron scattering [34-36]. Both results indicate that the scattering pattern does not reflect the instantaneous electron density. Rather, it appears as if the dynamics of the scattering pattern reflects the instantaneous momentum distribution of the electrons. Hence, it remains an open question whether time-resolved $\mathrm{x}$-ray scattering, in the quasielastic regime, can be used to 
image electron motion. An alternative, which allows imaging of the instantaneous electron density, is time-resolved phase contrast imaging [37]. However, it will be challenging to develop detector pixels that are small enough to resolve the image of small objects such as molecules.

Time-resolved Compton scattering has been discussed within the framework of the Holstein model, but without a theoretical analysis of the underlying ultrafast $x$-ray scattering process [38]. It has also been proposed as a means to characterize ultrashort $\mathrm{x}$-ray pulses via a Compton attosecond streak camera [39]. Theoretical investigations have been carried out by Shao et al. for the case of electron scattering [34-36]. In their work, they developed theories for $(e, 2 e)$ spectroscopy [34], as well as for energy-resolved electron scattering [36]. They applied their results to the scattering from coherent superpositions of hydrogen orbitals.

Here, we propose momentum-space imaging as an alternative to the approaches mentioned above. It is known that the scattering cross section for Compton scattering from a stationary sample gives access to the electron momentum distribution [40-43]. Therefore, we develop a QED-based theory of time-resolved Compton scattering. We find an expression for the doubly differential scattering cross section that is determined only by the instantaneous electron momentum density. This suggests the possibility to image electron wave-packet dynamics via time-resolved Compton scattering. One only has to keep in mind that one no longer gets information about the real-space electron density but rather about the momentum-space electron density.

To investigate the feasibility of our proposal, we apply our theory to electron wave-packet dynamics in argon atoms in a pump-probe setup. Unlike the previous works on time-resolved scattering, we do not restrict our investigation to superpositions of hydrogen-like orbitals. Rather, we consider a setup in which Ar is irradiated by up to two extreme ultraviolet (XUV) pump pulses. The resulting particle-hole wave packet is then probed by x-ray scattering. The first pump pulse excites or ionizes an electron, mostly from the $3 p$ shell. This gives us the opportunity to study the ultrafast scattering signal for the wave packet formed by the ionized electron. A second pulse may create a coherent superposition of the $3 s$ hole and the $3 p_{0}$ hole in the remaining ion. This allows us to study the imaging of coherent hole dynamics in the remaining ion.

To simulate the complex electron dynamics, we use the time-dependent configuration-interaction singles method (TDCIS) [44,45]. This is an ab initio electronic-structure method. It has been applied successfully to a variety of processes, such as high-harmonic generation [44,46], photoabsorption spectroscopy $[47,48]$, and optical strong-field processes [49,50]. In TDCIS, the wave function is restricted to single-particle single-hole excitations relative to the HartreeFock ground state. Moreover, the full nonrelativistic electronic Hamiltonian is kept, in order to capture electron-correlation dynamics.

The structure of the article is as follows: In Sec. II, we present our theory of time-resolved Compton scattering. Section III contains a brief introduction to the TDCIS method. Moreover, it contains details about constructing the electron instantaneous momentum density, and we discuss how this is used to calculate scattering cross sections. In Sec. IV, we apply our theory to investigate electron dynamics in Ar. The first part of the section is devoted to the dynamics of the wave packet created by the first pulse. In the second part of the section, we consider the dynamics of the hole wave packet created by a sequence of two XUV pulses. Finally, we summarize our results and give a conclusion in Sec. V.

\section{THEORY OF TIME-RESOLVED COMPTON SCATTERING}

\section{A. Formalism}

In a typical light-matter scattering setup, the system of interest can be split into an electronic part and the radiation field. The dynamics of the electronic system are governed by the Hamiltonian $\hat{H}_{\text {matter. }}$. It contains the electron kinetic energy as well as their attraction by atomic nuclei and the interaction between electrons. For more complex systems, such as molecules, one also needs to consider the nuclear dynamics, which are generally coupled to the electron dynamics. To describe the electronic part of the system, we shall employ the formalism of second quantization [51-53]. Electrons can then be viewed as excitations of a quantum field $\hat{\psi}_{\sigma}$. The corresponding field operators $\hat{\psi}_{\sigma}^{\dagger}(\mathbf{x})$ and $\hat{\psi}_{\sigma}(\mathbf{x})$, respectively, create and annihilate an electron with spin $\sigma$ at position $\mathbf{x}$. They satisfy well-known fermionic anticommutator relations [51].

The dynamics of the radiation field are governed by the Hamiltonian $\hat{H}_{\text {rad }}$. Excitations of its eigenmodes are called photons. They can be described by using a set of annihilation and creation operators $\hat{a}_{\mathbf{k}, \lambda}$ and $\hat{a}_{\mathbf{k}, \lambda}^{\dagger}$ fulfilling bosonic commutator relations $[51,54,55]$. Applying $\hat{a}_{\mathbf{k}, \lambda}^{\dagger}\left(\hat{a}_{\mathbf{k}, \lambda}\right)$ adds (removes) a photon with polarization vector $\boldsymbol{\epsilon}_{\lambda}$ and wave vector $\mathbf{k}$ to (from) the radiation field. To describe an arbitrary pure state of the radiation field, the former can be decomposed into a superposition of multimode Fock states $\left|\left\{n_{\mathbf{k}, \lambda}\right\}\right\rangle$. The latter are eigenstates of the photon-number operators $\hat{n}_{\mathbf{k}, \lambda}=\hat{a}_{\mathbf{k}, \lambda}^{\dagger} \hat{a}_{\mathbf{k}, \lambda}$, and thus of $\hat{H}_{\text {rad }}$. They are generated through the action of the creation operators on the vacuum state $|0\rangle$.

In the following derivation, we will be using the Coulomb gauge $(\boldsymbol{\nabla} \cdot \mathbf{A}=0)$. Also, atomic units $\left(\hbar=c=m_{e}=e=1\right)$ shall be employed. When using the principle of minimal coupling, the interaction between the electron field and the radiation field takes the form [56]

$$
\begin{aligned}
\hat{H}_{\text {int }}= & \alpha \int d^{3} x \hat{\psi}^{\dagger}(\mathbf{x})\left[\hat{\mathbf{A}}(\mathbf{x}) \cdot \frac{\nabla}{i}\right] \hat{\psi}(\mathbf{x}) \\
& +\frac{\alpha^{2}}{2} \int d^{3} x \hat{\psi}^{\dagger}(\mathbf{x}) \hat{\mathbf{A}}^{2}(\mathbf{x}) \hat{\psi}(\mathbf{x}) .
\end{aligned}
$$

The first term (the $\mathbf{p} \cdot \mathbf{A}$ term) can describe scattering only in second-order perturbation theory or higher. It is negligible for photon energies much larger than all inner-shell thresholds of the electronic system of interest. Since we are interested in the case of nonresonant scattering, we shall only consider the second term (the $\mathbf{A}^{2}$ term), which can induce scattering in first-order perturbation theory. Here, the scattering event is described as the simultaneous absorption and emission of a photon at a single interaction vertex. Further simplification is achieved by making use of the interaction picture. As a consequence, the dynamics of states are driven solely by $\hat{H}_{\text {int }}$. 
Generally speaking, calculating the scattering cross section means calculating the expectation value of a suitably chosen observable $\hat{\mathcal{O}}$. Let us write the initial state of the system as a density matrix $\hat{\rho}_{\text {in }}=\hat{\rho}_{\text {in }}^{X} \otimes \hat{\rho}_{\text {in }}^{\text {el }}$, where $\hat{\rho}_{\text {in }}^{X}$ is the initial density matrix of the radiation field, and $\hat{\rho}_{\mathrm{in}}^{\mathrm{el}}$ is the density matrix describing the initial state of the electronic system. We calculate the time evolution of $\hat{\rho}_{\text {in }}$ in first-order perturbation theory with respect to $H_{\text {int }}$. This leads to the following expression for the expectation value of an observable in the interaction picture:

$$
\begin{aligned}
\langle\hat{\mathcal{O}}\rangle_{t}= & \operatorname{Tr}[\hat{\rho}(t) \hat{\mathcal{O}}(t)] \\
= & \operatorname{Tr}\left[\hat{\rho}_{\text {in }} \hat{\mathcal{O}}(t)\right]+2 \operatorname{Re}\left\{i \int_{-\infty}^{t} d t^{\prime} \operatorname{Tr}\left[\hat{\rho}_{\text {in }} \hat{H}_{\text {int }}\left(t^{\prime}\right) \hat{\mathcal{O}}(t)\right]\right\} \\
& +\int_{-\infty}^{t} d t^{\prime} \int_{-\infty}^{t} d t^{\prime \prime} \operatorname{Tr}\left[\hat{H}_{\text {int }}\left(t^{\prime}\right) \hat{\rho}_{\text {in }} \hat{H}_{\text {int }}\left(t^{\prime \prime}\right) \hat{\mathcal{O}}(t)\right] .
\end{aligned}
$$

If $\hat{\mathcal{O}}$ describes the intensity of photons hitting a detector, there is a simple interpretation of the different terms. The first term describes the incoming, unscattered radiation, whereas the last term [the $(1,1)$ term] describes the scattered radiation. The second term [the $(0,1)$ term] describes interference between the scattered and the incoming photons. It can be shown that this interference term is negligible in the far-field regime. However, it does play an important role in the near-field regime. Specifically, it gives rise to the signal used in phase-contrast imaging $[37,57]$. On the other hand, the far-field scattering pattern is determined by the last term. Finally, note the special case that one considers an initial state $\hat{\rho}_{\text {in }}=|I\rangle\langle I|$ and an observable that is a projection operator onto some final state $|F\rangle$. Then, for $t \rightarrow \infty$ the $(1,1)$ term gives rise to a transition rate from $|I\rangle$ to $|F\rangle$ that reproduces Fermi's golden rule.

\section{B. Scattering pattern}

The intensity of scattered radiation can be found by calculating the $(1,1)$ term of Eq. (3) for a suitable observable. Possible choices include the quantum-mechanical Poynting operator [55] and a projection operator onto the photon modes contributing to the scattering into a given solid angle. Let us assume that the incoming probe pulse has a small bandwidth, a small angular spread, and that all contributing modes have the same polarization. Moreover, we assume that the probe pulse is short compared with the timescale of the electron dynamics. Under these circumstances the differential scattering cross section takes the following form [30,58]:

$$
\begin{aligned}
\frac{d \sigma}{d \Omega}= & \frac{d \sigma_{\mathrm{th}}}{d \Omega} \sum_{f} \int_{\omega_{\min }}^{\omega_{\max }} d \omega_{\mathbf{k}_{s}} \frac{\omega_{\mathbf{k}_{s}}}{\omega_{\mathrm{in}}} W_{1}\left(\omega_{\mathrm{in}}-\omega_{\mathbf{k}_{s}}+E_{\mathrm{el}}-E_{f}\right) \\
& \times\left|\int d^{3} x\left\langle\Psi_{f}|\hat{n}(\mathbf{x})| \Psi_{\mathrm{el}}\left(\tau_{\text {delay }}\right)\right\rangle e^{i \mathbf{Q} \cdot \mathbf{x}}\right|^{2}
\end{aligned}
$$

Here, the electronic system is assumed to be a pure-state coherent wave packet $\left|\Psi_{\mathrm{el}}\right\rangle\left\langle\Psi_{\mathrm{el}}\right|$ and $\tau_{\text {delay }}$ is the time delay at which the x-ray pulse interacts with the electronic wave packet. $E_{\mathrm{el}}$ is the energy of the electronic wave packet, and $E_{f}$ is the energy of the final electronic state $\left|\Psi_{f}\right\rangle$. The sum extends over all accessible, final electronic states. The values $\omega_{\min }$ and $\omega_{\max }$, respectively, describe the minimum and maximum photon energy accepted by the x-ray detector. Finally, we introduced the function $W_{1}(\omega)=\frac{T_{R}}{\sqrt{8 \pi \ln 2}} e^{-T_{R}^{2} \omega^{2} /(8 \ln 2)} . W_{1}$ is the Fourier transform of the first-order correlation function of the incoming x-ray pulse. Here, the pulse is modeled as a coherent pulse. The full width at half maximum of the envelope of the temporal intensity profile is given by $T_{R}$.

In the following, let us consider the triply differential scattering cross section:

$$
\begin{aligned}
\frac{d^{3} \sigma_{c}}{d \Omega d \omega_{\mathbf{k}_{s}} d \Omega_{e}}= & \frac{d \sigma_{\mathrm{th}}}{d \Omega} \frac{\omega_{\mathbf{k}_{s}}}{\omega_{\mathrm{in}}} \sum_{f \mid \Omega_{e}} W_{1}\left(\omega+E_{\mathrm{el}}-E_{f}\right) \\
& \times\left|\int d^{3} x\left\langle\Psi_{f}|\hat{n}(\mathbf{x})| \Psi_{\mathrm{el}}\left(\tau_{\text {delay }}\right)\right\rangle e^{i \mathbf{Q} \cdot \mathbf{x}}\right|^{2},
\end{aligned}
$$

where we introduced the energy transfer from the radiation field to the recoil electron $\omega=\omega_{\text {in }}-\omega_{\mathbf{k}_{s}}$. It can be obtained from Eq. (4) by restricting the sum over final states to those where a recoil electron is emitted into the solid angle $d \Omega_{e}$ and by dropping the integration over final photon energies. In the following, we assume that the electronic system, described by $\left|\Psi_{\text {el }}\left(\tau_{\text {delay }}\right)\right\rangle$, is in a state with a total spin $S \equiv 0$.

Rather than employing the impulse approximation, in order to further simplify the expression, we extend the approach of Ref. [59] to the time-dependent case. We start by employing the sudden approximation. This means that we assume the final state to be a product state of recoil electron and remaining ion $\left|\Psi_{f}\right\rangle \approx \hat{c}_{\epsilon, \sigma}^{\dagger}\left|\Psi_{n, \bar{\sigma}}^{N-1}\right\rangle$. Here, $\hat{c}_{\epsilon, \sigma}^{\dagger}$ creates an unbound recoil electron in the potential of the ion, with kinetic energy $\epsilon$, spin $\sigma \in\{\uparrow, \downarrow\}$, and propagating in the direction of $\Omega_{e}$. The remaining ion is left in the $(N-1)$-electron state $\left|\Psi_{n, \bar{\sigma}}^{N-1}\right\rangle$, where $n$ characterizes the excitation of the ion, and $\bar{\sigma}$ is the spin opposite to $\sigma$. Making this approximation implies that we are neglecting any correlations between the recoil electron and the remaining $(N-1)$-electron system. This is justified if the kinetic energy of the electron is large compared with the valence binding energies of the system. By inserting our ansatz for the final states, we obtain

$$
\begin{aligned}
& \frac{d^{3} \sigma_{c}}{d \Omega d \omega_{\mathbf{k}_{s}} d \Omega_{e}} \\
& =\frac{d \sigma_{\text {th }}}{d \Omega} \frac{\omega_{\mathbf{k}_{s}}}{\omega_{\text {in }}} \sum_{n, \epsilon, \sigma} W_{1}\left(\omega-\epsilon+E_{\mathrm{el}}-E_{n}^{N-1}\right) \\
& \quad \times\left|\int d^{3} x\left\langle\Psi_{n, \bar{\sigma}}^{N-1}\left|\hat{c}_{\epsilon, \sigma} \hat{\psi}^{\dagger}(\mathbf{x}) \hat{\psi}(\mathbf{x})\right| \Psi_{\text {el }}\left(\tau_{\text {delay }}\right)\right\rangle e^{i \mathbf{Q} \cdot \mathbf{x}}\right|^{2} .
\end{aligned}
$$

Our approximation is justified if the recoil electron leaves the system very quickly. Therefore its kinetic energy has to be much larger than $\left|E_{\mathrm{el}}-E_{n}^{N-1}\right|$, and the corresponding term can be neglected in the argument of $W_{1}$.

Next we expand $\hat{\psi}(\mathbf{x})$ in a basis of one-electron states $\left\{\varphi_{q, \sigma}\right\}$ :

$$
\hat{\psi}(\mathbf{x})=\sum_{q, \sigma} \varphi_{q, \sigma}(\mathbf{x}) \hat{c}_{q, \sigma} .
$$

Within our model, the state of the recoil electron is not occupied in the initial wave packet, i.e., $\hat{c}_{\epsilon, \sigma}\left|\Psi_{\text {el }}\left(\tau_{\text {delay }}\right)\right\rangle=0$. In consequence, the matrix elements in Eq. (6) are only nonzero if the field operator $\hat{\psi}^{\dagger}(\mathbf{x})$ creates an electron in the corresponding state. $\hat{\psi}^{\dagger}(\mathbf{x})$ can then be replaced by $\varphi_{\epsilon, \sigma}^{\dagger}(\mathbf{x}) \hat{c}_{\epsilon, \sigma}^{\dagger}$, 
and $\left\langle\Psi_{n, \bar{\sigma}}^{N-1}\right| \hat{\epsilon}_{\epsilon, \sigma} \hat{\psi}^{\dagger}(\mathbf{x})=\varphi_{\epsilon, \sigma}^{\dagger}(\mathbf{x})\left\langle\Psi_{n, \bar{\sigma}}^{N-1}\right|$. Thus, using the timedependent Dyson orbitals

$$
g_{n, \sigma}\left(\mathbf{x}, \tau_{\text {delay }}\right)=\sum_{q} \varphi_{q, \sigma}(\mathbf{x})\left\langle\Psi_{n, \bar{\sigma}}^{N-1}\left|\hat{c}_{q, \sigma}\right| \Psi_{\text {el }}\left(\tau_{\text {delay }}\right)\right\rangle,
$$

we obtain

$$
\begin{aligned}
\frac{d^{3} \sigma_{c}}{d \Omega d \omega_{\mathbf{k}_{s}} d \Omega_{e}}= & \frac{d \sigma_{\mathrm{th}}}{d \Omega} \frac{\omega_{\mathbf{k}_{s}}}{\omega_{\text {in }}} \sum_{n, \epsilon, \sigma} W_{1}(\omega-\epsilon) \\
& \times\left|\int d^{3} x \varphi_{\epsilon, \sigma}^{\dagger}(\mathbf{x}) g_{n, \sigma}\left(\mathbf{x}, \tau_{\text {delay }}\right) e^{i \mathbf{Q} \cdot \mathbf{x}}\right|^{2} .
\end{aligned}
$$

Finally, we make the assumption that the recoil electron can be described by a plane wave,

$$
\varphi_{\epsilon, \sigma}=\frac{1}{\sqrt{V}} \exp \left(i \mathbf{p}_{\epsilon} \cdot \mathbf{x}\right) \chi_{\sigma} .
$$

Here, $\chi_{\sigma}$ is a two-component spinor with spin $\sigma$. Now, the spatial integral in Eq. (9) just yields the Fourier transform $\chi_{\sigma}^{\dagger} \tilde{g}_{n, \sigma}\left(\mathbf{Q}-\mathbf{p}_{e}, \tau_{\text {delay }}\right)$ of the Dyson orbitals. Keeping in mind that the $(N-1)$-electron states form a complete set, it follows that

$$
\sum_{n, \sigma}\left|\tilde{g}_{n, \sigma}\left(\mathbf{P}, \tau_{\text {delay }}\right)\right|^{2}=\rho\left(\mathbf{P}, \tau_{\text {delay }}\right),
$$

where $\mathbf{P}:=\mathbf{Q}-\mathbf{p}_{e}$ is the momentum transferred to the ion and $\rho\left(\mathbf{P}, \tau_{\text {delay }}\right)$ is the instantaneous momentum density,

$$
\begin{aligned}
\rho\left(\mathbf{P}, \tau_{\text {delay }}\right)= & \sum_{p, q} \sum_{\sigma} \varphi_{p, \sigma}^{\dagger}(\mathbf{P}) \varphi_{q, \sigma}(\mathbf{P}) \\
& \times\left\langle\Psi_{\text {el }}\left(\tau_{\text {delay }}\right)\left|\hat{c}_{p, \sigma}^{\dagger} \hat{c}_{q, \sigma}\right| \Psi_{\text {el }}\left(\tau_{\text {delay }}\right)\right\rangle,
\end{aligned}
$$

of the electronic wave packet. Combining the previous results and replacing $\sum_{\epsilon}$ by $\frac{V}{(2 \pi)^{3}} \int d \epsilon \sqrt{2 \epsilon}$ yields

$$
\begin{aligned}
& \frac{d^{3} \sigma_{c}}{d \Omega d \omega_{\mathbf{k}_{s}} d \Omega_{e}} \\
& =\frac{d \sigma_{\text {th }}}{d \Omega} \frac{\omega_{\mathbf{k}_{s}}}{\omega_{\text {in }}} \int d \epsilon \sqrt{2 \epsilon} W_{1}(\omega-\epsilon) \rho\left(\mathbf{Q}-\mathbf{p}_{\epsilon}, \tau_{\text {delay }}\right) .
\end{aligned}
$$

The doubly differential scattering cross section (DDSCS) can be obtained by integrating over $\Omega_{e}$ :

$$
\begin{aligned}
\frac{d^{2} \sigma_{c}}{d \Omega d \omega_{\mathbf{k}_{s}}}= & \frac{d \sigma_{\text {th }}}{d \Omega} \frac{\omega_{\mathbf{k}_{s}}}{\omega_{\text {in }}} \int d^{3} P \\
& \times W_{1}\left(\omega-\frac{P^{2}}{2}-\frac{Q^{2}}{2}+\mathbf{P} \cdot \mathbf{Q}\right) \rho\left(\mathbf{P}, \tau_{\text {delay }}\right) .
\end{aligned}
$$

Remarkably, we find that the scattering pattern depends simply on the instantaneous momentum density of the electronic wave packet. The finite pulse duration only manifests itself in the function $W_{1}$. Note that, when taking the limit of a long pulse $\left(T_{R} \rightarrow \infty\right)$ and assuming a stationary electronic state for $\left|\Psi_{\text {el }}\left(\tau_{\text {delay }}\right)\right\rangle$, one recovers the usual expression for the Compton profile in the impulse approximation [59,60]. Thus, we can conclude that, under the assumptions made, the usual picture of Compton scattering from free electrons with a given momentum distribution $\rho(\mathbf{P})$ can still be applied. However, in the familiar monochromatic case, the momenta contributing to the scattering pattern are restricted to a two-dimensional manifold of momentum space. This is caused by energy conservation. In the case of short pulses, the incident photons have various energies determined by the spectral bandwidth of the pulse. Therefore, all momenta contribute to the scattering cross section. The strength of their contribution is determined by the Gaussian function $W_{1}\left(\omega-\frac{P^{2}}{2}-\frac{Q^{2}}{2}+\mathbf{P} \cdot \mathbf{Q}\right)$, which generalizes the energy-conserving term $\frac{1}{Q} \delta\left(P_{\|}+\frac{\omega}{Q}-\frac{Q}{2}\right)$, known from the monochromatic case. Here, $P_{\|}$refers to the component of $\mathbf{P}$ that is parallel to $\mathbf{Q}$. The bandwidth of the pulse is determined by its pulse duration $T_{R}$.

Equation (14) is particularly remarkable when placed in the context of previous work on time-resolved scattering [30,61]. For elastic scattering with a long pulse from a stationary electronic state, the scattering pattern gives access to the modulus squared of the Fourier transform of the electron density. However, this picture breaks down for short pulses. For wave packets in hydrogen it seems like the scattering patterns reflect the electron instantaneous momentum density instead of their real-space density $[30,35,36]$. This apparent connection between the instantaneous momentum density and the scattering pattern can be understood in terms of our result for time-resolved Compton scattering.

It should be stressed that there is a distinct difference between the Fourier picture in coherent scattering and the momentum-space picture in Compton scattering. One cannot obtain the real-space density by Fourier transforming the momentum density. A simple calculation confirms that the Fourier transformation of the momentum density yields a spatial autocorrelation function rather than the real-space density.

Finally, a comment should be made about energy resolution. Currently, detectors for imaging experiments at XFEL facilities are designed to measure photons without energy resolution [62-64]. Therefore, the accessible quantity is the differential scattering cross section rather than the doubly differential scattering cross section. This quantity can be obtained, when integrating the DDSCS over the energies $\omega_{\mathbf{k}_{s}}$ of the scattered photons. It can be computed very efficiently when using the Waller-Hartree approximation [65]. In other words, one assumes $\omega_{\mathbf{k}_{s}} \approx \omega_{\text {in }}$. Under those conditions, the relationship between momentum transfer and scattering angle is the same as in elastic scattering. This approximation is valid whenever the energy transfer is small compared with $\omega_{\text {in }}$. It has been applied successfully for calculating inelastic structure factors of various atoms and molecules. Moreover, recent studies of the static structure factor of carbon show very good agreement between the result of a direct integration and that obtained by using the Waller-Hartree approach [66]. Let us assume that the detector measures all the scattered photons with energies in the interval $\left[\omega_{\min }, \omega_{\max }\right]$. Carrying out the integral over $\omega_{\mathbf{k}_{s}}$, the Compton-scattering contribution to the differential scattering cross section becomes

$$
\begin{aligned}
\frac{d \sigma_{c}}{d \Omega}= & \frac{1}{2} \frac{d \sigma_{\text {th }}}{d \Omega} \int d^{3} P\left[\operatorname{erf}\left(\frac{T_{R}}{\sqrt{8 \ln 2}} \tilde{\omega}_{\text {max }}\right)\right. \\
& \left.-\operatorname{erf}\left(\frac{T_{R}}{8 \ln 2} \tilde{\omega}_{\text {min }}\right)\right] \rho\left(\mathbf{P}, \tau_{\text {delay }}\right) .
\end{aligned}
$$

Here, $\operatorname{erf}(\mathrm{x})$ is the error function, and $\tilde{\omega}_{\min , \max }=\omega_{\min , \max }-$ $\frac{1}{2}(\mathbf{P}-\mathbf{Q})^{2}$. Now, let us consider the limit in which the detectors have no energy resolution. In that case, one can 
assume that $\frac{T_{R}}{\sqrt{2 \ln 2}}\left|\tilde{\omega}_{\min , \max }\right| \gg 1$. As a consequence, the error functions in Eq. (15) become constant for all relevant $\mathbf{P}$ and Q. In consequence, one is left with an integral over the electron instantaneous momentum density. In this limit, the differential Compton-scattering cross section is determined solely by the number of electrons. Since this is a conserved quantity, the scattering signal due to Compton scattering is no longer time dependent. This might appear surprising in the light of the results of Ref. [58], where it was found that, even without energy resolution, dynamic imaging should be possible because the scattering probability still depends on the time-dependent electron pair-correlation function. However, this is no contradiction because our derivation only considers those contributions in which an electron is scattered into a state that can be modeled as a plane wave. The remaining contributions to the scattering pattern still depend on $\tau_{\text {delay }}$. Those also include contributions due to coherent scattering. For sufficiently small values of $\mathbf{Q}$, this contribution is expected to become dominant because it grows quadratically with the number of electrons. On the other hand, Compton scattering is dominant for large values of the momentum transfer.

The results obtained here rely on the fact that we model the final state of the recoil electron as a plane-wave state. This implies that the state of the recoil electron is assumed to be independent of the parent ion. Strictly speaking, this picture is only valid if the kinetic energy of the recoil electron, after scattering, is much larger than its binding energy, before the scattering. Thus one requires large energy transfers or, equivalently, $\frac{\left\langle p_{i}\right\rangle}{Q} \ll 1$. Here, $\left\langle p_{i}\right\rangle$ is the mean momentum of an electron in the orbital $\left|\varphi_{i}\right\rangle$. This is a strong assumption, and it cannot be expected that it is always fulfilled. In the case that it is not, it is no longer clear that the differential Compton-scattering section should be stationary. For stationary targets comparisons of theory and experiment have been done [43]. These show that the impulse approximation still yields satisfactory results for $\frac{\left\langle p_{i}\right\rangle}{Q} \lesssim 1$. In consequence, Eqs. (14) and (15) should yield good results for the scattering of $\mathrm{x}$-ray photons from valence electrons and from free electrons. When the assumptions made are valid, our results are useful for time-resolved imaging studies. Consider the case that only valence electrons undergo dynamics while inner-shell electrons remain stationary. In that case, changes in the scattering pattern are only caused by photons that scatter from valence electrons or from excited electrons. If we focus on those electrons, our assumptions should be well fulfilled except for very small photon energy transfers. In an experiment, the relevant signal could be extracted by first measuring a scattering pattern when the system has not yet been excited. By only considering the changes in the subsequent scattering patterns, one eliminates the contributions from scattering by the strongly bound, stationary electrons.

\section{COMPUTATIONAL METHOD}

\section{A. Time-dependent configuration-interaction singles method}

To simulate the dynamics of the electronic system, we use the time-dependent configuration-interaction singles method (TDCIS) [44,45]. It has been applied successfully in the investigation of many ultrafast processes in intense light pulses $[29,44,46,47,67]$. In this approach, the wave function of the many-body system is expanded in terms of the Hartree-Fock ground state of the system $\left|\Phi_{0}\right\rangle$ and 1-particle-1-hole $(1 \mathrm{p} 1 \mathrm{~h})$ excitations. The latter are formed by acting with one creation and one annihilation operator on the Hartree-Fock ground state:

$$
\left|\Phi_{i}^{a}\right\rangle=\hat{c}_{a}^{\dagger} \hat{c}_{i}\left|\Phi_{0}\right\rangle
$$

Here and in the following, indices $i, j$ refer to spin orbitals that are occupied in $\left|\Phi_{0}\right\rangle$, whereas $a, b$ refer to orbitals that are not occupied. Using time-dependent coefficients, the many-body wave function is written as

$$
|\Psi(t)\rangle=\alpha_{0}(t)\left|\Phi_{0}\right\rangle+\sum_{i, a} \alpha_{i}^{a}(t)\left|\Phi_{i}^{a}\right\rangle .
$$

This comparably simple ansatz already captures a great deal of the electronic correlations and excitations. This can be concluded from the good agreement between theoretical and experimental results (see also Ref. [48]). Note that TDCIS does not capture many-electron processes such as the Auger decay of a $1 \mathrm{p} 1 \mathrm{~h}$ excitation. This could be achieved by including higher-order excitations in the ansatz for the wave function. However, a larger basis set increases the numerical effort necessary to solve the problem. Besides that, there are other conceptual advantages to configuration-interaction singles. First, the Brillouin theorem assures that the Hamiltonian of the electronic system is block diagonal with respect to the Hartree-Fock ground state and the $1 \mathrm{p} 1 \mathrm{~h}$ states. Thus, any coupling between $\left|\Phi_{0}\right\rangle$ and a $1 \mathrm{p} 1 \mathrm{~h}$ state is due to the interaction with the light field. Second, it allows for the very intuitive picture that the hole index $i$ corresponds to the ionic eigenstate, making use of Koopmans' theorem. This picture is not valid in the case of higher-order configuration interaction [68]. Within the TDCIS framework, the expectation value of one-particle operators, such as the electron density, can be calculated very easily. Also, one has access to the ionic density matrix $[29,44]$. This allows us to investigate the degree of coherence between different ionic channels.

The Hamiltonian of the electronic system is given by

$$
\hat{H}(t)=\hat{F}+\hat{V}_{C}-\hat{V}_{\mathrm{HF}}-E_{\mathrm{HF}}-\mathcal{E}(t) \hat{z} .
$$

Here, $\hat{F}$ is the Fock operator, which fulfills $\hat{F}\left|\varphi_{p, \sigma}\right\rangle=$ $\varepsilon_{p}\left|\varphi_{p, \sigma}\right\rangle . \hat{V}_{C}$ is the Coulomb interaction between electrons, $\hat{V}_{\mathrm{HF}}$ is the Hartree-Fock mean-field potential, and $E_{\mathrm{HF}}$ is the Hartree-Fock ground-state energy. Finally, $\hat{z}$ is the dipole operator, and $\mathcal{E}(t)$ is the electric-field component of the probe pulse. Note that we neglect spin-orbit coupling. The interaction between electrons and the radiation field is now described in the length gauge. Also, we assume the pump pulse to be polarized along the $z$ direction, and we make use of the dipole approximation. The result for the wave packet might change slightly if it was calculated in velocity gauge. This is due to the fact that the $\mathbf{p} \cdot \mathbf{A}$ operator can couple to higher excited states than the dipole operator in length gauge [45]. Note that this choice of gauge is not a problem as long as we consider the probe process separately from the pump step. This is valid as long as the temporal overlap between pump and probe pulse is small. In that case, one can simply gauge transform the electronic wave packet into the velocity gauge. It is clear that such a transformation leaves the 
scattering cross section invariant because it changes neither the instantaneous momentum density nor the transition matrix elements $\left\langle\varphi_{k}|\hat{n}(\mathbf{x})| \varphi_{l}\right\rangle$.

By projecting the Schrödinger equation onto the HartreeFock ground state and onto the $1 \mathrm{p} 1 \mathrm{~h}$ states, one obtains the following equations of motion for the time-dependent coefficients [44]:

$$
\begin{gathered}
i \dot{\alpha}_{0}(t)=-\sqrt{2} \mathcal{E}(t) \sum_{i, a} \alpha_{i}^{a}(t) z_{i, a} \\
i \dot{\alpha}_{i}^{a}(t)=\left(\varepsilon_{a}-\varepsilon_{i}\right) \alpha_{i}^{a}(t)+\sum_{i^{\prime}, a^{\prime}} \alpha_{i^{\prime}}^{a^{\prime}}(t)\left(2 v_{a, i^{\prime}, i, a^{\prime}}-v_{a, i^{\prime}, a^{\prime}, i}\right) \\
-\mathcal{E}(t)\left[\sqrt{2} \alpha_{0}(t) z_{a, i}+\sum_{a^{\prime}} \alpha_{i}^{a^{\prime}}(t) z_{a, a^{\prime}}-\sum_{i^{\prime}} \alpha_{i^{\prime}}^{a}(t) z_{i^{\prime}, i}\right] .
\end{gathered}
$$

Here, all indices refer to purely spatial orbitals. The matrix elements are defined as $z_{p, q}=\left\langle\varphi_{p}|\hat{z}| \varphi_{q}\right\rangle$, and $v_{p, q, r, s}=$ $\left\langle\varphi_{p} \varphi_{q}\left|1 / \hat{r}_{12}\right| \varphi_{r} \varphi_{s}\right\rangle$. The Hartree-Fock orbitals are written as a product of spherical harmonics $Y_{l, m}\left(\theta_{r}, \phi_{r}\right)$ and a radial function $R_{n, l}(r)=\frac{u_{n, l}(r)}{r}$. To adequately describe the problem, one needs a radial grid with sufficient density near the atomic nucleus $(r=0)$ as well as far away from the nucleus. Hence, the calculations were carried out using primitive points on a Gauss-Lobatto grid, which were then mapped to the actual grid. Details can be found in Ref. [44]. For our calculations, the mapping parameters were $\zeta=1.0$ and $L=75.0$, such that $r \in(0.0,150.0) a_{0}$.

\section{B. Calculation of the Compton-scattering cross section}

After solving the TDCIS equations of motion, the instantaneous momentum density can be constructed. To do so, one first needs to find the momentum-space representation of the Hartree-Fock orbitals. By performing the Fourier transform, the following result is obtained for an orbital with quantum numbers $n, l$, and $m$ :

$$
\begin{aligned}
\varphi_{n, l, m}\left(p, \theta_{p}, \phi_{p}\right)= & -\sqrt{\frac{2}{\pi}}(-i)^{l} Y_{l, m}\left(\theta_{p}, \phi_{p}\right) \\
& \times \int_{0}^{\infty} d r r u_{n, l}(r) j_{l}(r p),
\end{aligned}
$$

where $j_{l}(x)$ is a spherical Bessel function of the first kind and $Y_{l, m}\left(\theta_{p}, \phi_{p}\right)$ is a spherical harmonic [69].

The calculation of the radial integrals in Eq. (21) is challenging. Strongly bound orbitals, such as the $1 s$ orbital, are very delocalized in momentum space. However, at high momenta, the spherical Bessel functions become more and more oscillatory. Therefore, a large number of radial grid points is needed to assure convergence. On the other hand, this large number of grid points slows down the TDCIS calculations considerably. Therefore, the TDCIS equations of motion were solved by using 1000 radial grid points. Then, the orbitals were interpolated to a suitable integration grid by using cubic splines.

Another important aspect for the convergence of the Compton-scattering signal is an adequate choice of grid points in momentum space. Unoccupied electron states have a peaked structure in momentum space. However, they also display some oscillations. These stem from the fact that one is taking the Fourier transform of a function on a finite-size grid. To keep the orbitals normalized, it is necessary to choose a momentum-space grid that is dense enough to capture the aforementioned oscillations. The period of those oscillations could be reduced by increasing the grid size, thus improving the resolution in momentum space. However, when increasing the grid size, one needs to increase the number of spatial grid points as well. Moreover, this would increase the number of orbitals that one has to consider. To check if our resolution in momentum space is sufficient, we did calculations on a grid with up to four times the extension of the original grid. These did not show any deviations in the momentum-space density. Hence, our resolution in momentum space appears to be good enough to adequately capture the dynamics of our system.

With the instantaneous momentum density at hand, we use Eqs. (14) and (15) to calculate the DDSCS and the differential scattering cross section, respectively. Note that our system of interest has cylindrical symmetry with respect to the pump polarization axis. Therefore, it is sufficient to sample the instantaneous momentum density at various $\theta_{p}$ and for one fixed value of $\phi_{p}$. However, note that we still need to compute the full three-dimensional integrals in Eqs. (14) and (15) numerically. There does not appear to be a simple way to factor out the integral over $\phi_{p}$. The angular integrals are carried out by using Simpson's rule. As done in the TDCIS implementation used here, the radial integrals are carried out by using the Lobatto quadrature scheme. For our calculations we used a uniform $\theta_{p}$ grid with 63 points. The integrals over $\phi_{p}$ were carried out by using 127 equidistant grid points.

\section{DYNAMICS OF Ar}

In this section, we apply our theory and investigate $\mathrm{x}$-ray imaging of electron dynamics in argon. Many earlier works on time-resolved scattering illustrated their results by using comparably simple systems [30,34,35,37,58,61,70]. Particularly popular choices are coherent superpositions of hydrogen orbitals. Depending on the symmetry of the orbitals, one obtains different dynamics (e.g., wiggling or breathing modes), which can then be probed by the scattering scheme at hand. There are some works, on resonant x-ray scattering, that deal with more complex systems, such as $\mathrm{Br}_{2},(\mathrm{KBr})_{108}$, and $(\mathrm{Ge})_{83}[71,72]$. In all earlier works on attosecond $\mathrm{x}$-ray scattering, the preparation of the initial states was not considered explicitly. In contrast, we explicitly consider the preparation of the electronic wave packet. Specifically, we apply our theory to argon atoms, where multichannel effects may be expected. To follow the electronic dynamics, under up to two pump pulses, we use the $a b$ initio method TDCIS.

\section{A. Results for one pump pulse}

By using the scheme described above, we investigate the dynamics of argon atoms after irradiation with a single attosecond XUV pulse centered at $t=0$. The mean photon energy of the pulse is $\omega_{1}=30 \mathrm{eV}$, and the pulse duration is 80 as. The peak electric-field strength of the pulse is $90 \mathrm{GV} / \mathrm{m}$. The electric field of the pulse is plotted in Fig. 1(b). The mean 

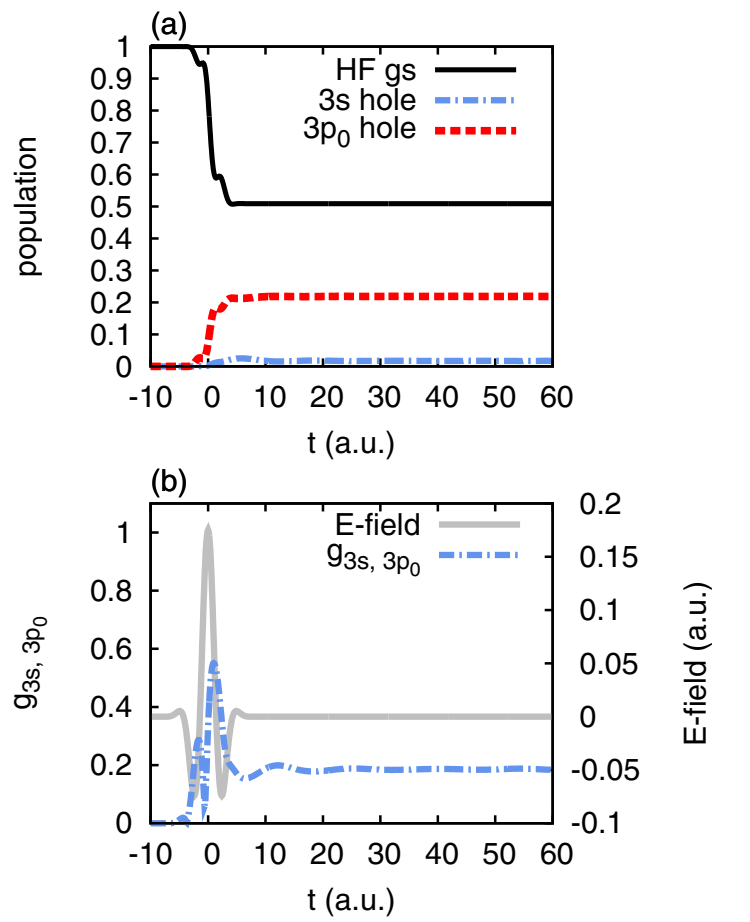

FIG. 1. Elements of the ionic density matrix for atomic Ar driven by an XUV pump pulse. Panel (a) shows the populations of the Hartree-Fock ground state (solid black line), the $3 \mathrm{~s}$ hole (dotteddashed blue line), and the $3 p_{0}$ hole (dashed red line), respectively. In panel (b) we show the degree of coherence between the $3 \mathrm{~s}$ and the $3 p_{0}$ holes (dotted-dashed blue line) and the pump electric field (solid gray line).

photon energy is high enough to ionize electrons from the $3 s$ shell $\left(\varepsilon_{3 s}=29.2 \mathrm{eV}\right)$ as well as from the $3 p$ shell $\left(\varepsilon_{3 p}=\right.$ $15.7 \mathrm{eV}$ ) [73]. Inner-shell electrons have a binding energy larger than $248 \mathrm{eV}$, which lies well beyond the bandwidth of the pulse. In Fig. 1(a), the hole populations for some valence orbitals are shown. One sees that ionization takes place primarily from the $3 p$ orbitals. The population of the $3 s$ hole is very small.

Figure 1(b) also shows the degree of coherence between the $3 s$ and $3 p_{0}$ holes. This quantity can be defined as [25,29,44]

$$
g_{i, j}(t)=\frac{\left|\rho_{i, j}^{\mathrm{IDM}}(t)\right|}{\sqrt{\rho_{i, i}^{\mathrm{IDM}}(t) \rho_{j, j}^{\mathrm{IDM}}(t)}},
$$

with $\rho_{i, j}^{\text {IDM }}$ being the elements of the ionic density matrix. One can see that hardly any coherence is built up between the $3 s$ and $3 p_{0}$ holes. In order for coherence to build up, the excited electrons of the $1 \mathrm{p} 1 \mathrm{~h}$ states need to be in the same oneparticle state. This can be achieved via multiphoton processes, as has been demonstrated by Goetz et al. [74,75]. For the large electric-field strengths considered here, it is possible that electrons with the same angular momentum are excited from both the $3 s$ and the $3 p_{0}$ orbital. However, in most cases they will have a different energy and therefore cannot contribute to building up coherence.

In Figs. 2(a)-2(d), we show the DDSCS at delay times $\tau_{\text {delay }}=6.9$ a.u., 10.9 a.u., 13.9 a.u., and 36.9 a.u., respectively.
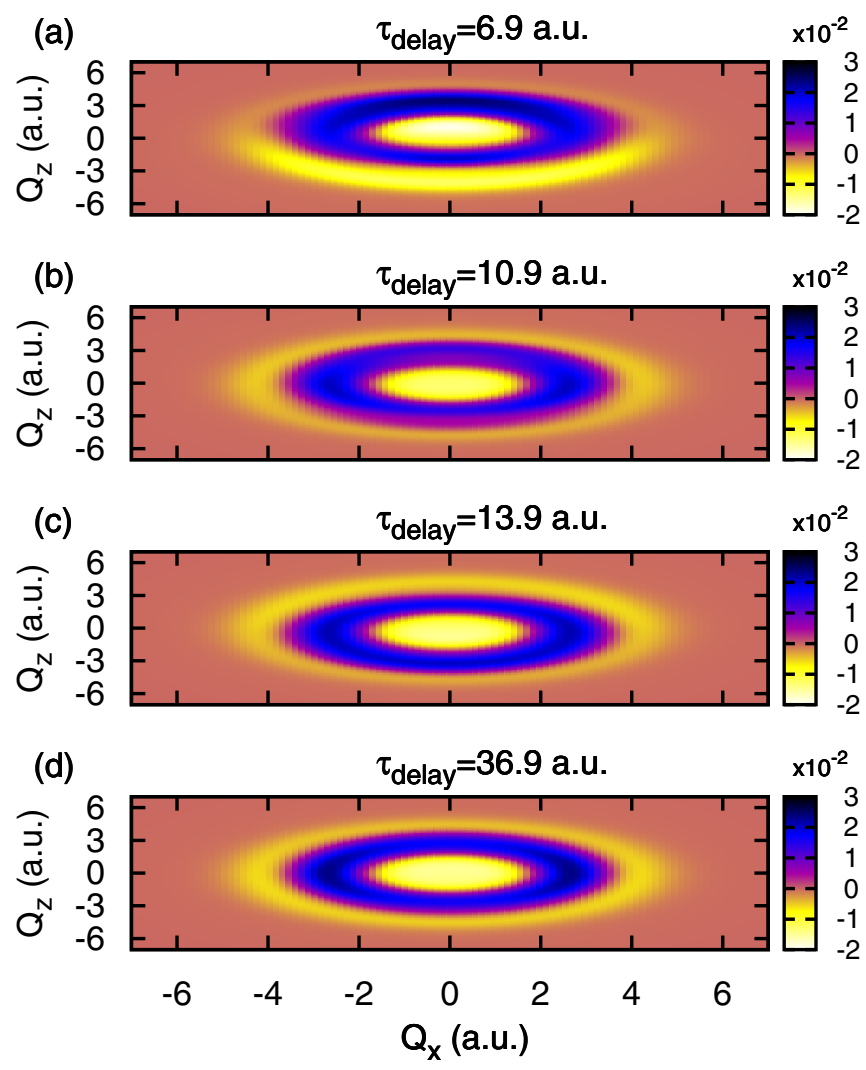

FIG. 2. DDSCS for energy transfer $\omega=100 \mathrm{eV}$ and $T_{R}=23.5$ as at delay times (a) $\tau_{\text {delay }}=6.9$ a.u., (b) 10.9 a.u., (c) 13.9 a.u., and (d) 36.9 a.u. in units of $d \sigma_{\text {th }} / d \Omega$. The $y$ component of the momentumtransfer vector is set to zero.

Here, the energy transfer was fixed to a value of $100 \mathrm{eV}$. The dynamics take place for momentum transfers smaller than 5 a.u. If one chooses an initial photon energy of $20 \mathrm{keV}$, one can safely assume that $\frac{\omega_{\mathbf{k} s}}{\omega_{\text {in }}} \approx 1$. The range of values for the momentum transfer then translates into scattering angles ranging from $0^{\circ}$ to $60^{\circ}$. It is desirable to have a small bandwidth of the probe pulse. In that way, for each $\mathbf{Q}$, the DDSCS probes only a small slice of the instantaneous momentum density. However, one must take care that the pulse remains short enough to freeze the electronic wave packet. Here, the probe pulse duration $T_{R}$ was fixed to a value of 23.5 as. For the results in this work, we assumed that the polarization of the probe pulse coincides with the $z$ direction. The dependence of the differential scattering cross section and the DDSCS on the wave vector of the incoming $x$-ray pulse is contained solely in the Thomson scattering cross section. The remaining factor, which contains the information about the electronic system, is invariant with respect to rotation about the $z$ axis. Moreover, we set $Q_{y}=0$. Thus, we display the DDSCS for a Euclidian plane in $\mathbf{Q}$ space. Typical scattering experiments usually probe more complicated manifolds, such as cuts through the Ewald sphere for the case of purely coherent scattering.

As mentioned in Sec. II B, the DDSCS was calculated by using the instantaneous momentum density with the contribution of the Hartree-Fock ground state subtracted. Hence, only changes in the DDSCS with respect to the ground state are shown. The scattering patterns all show a 
positive-valued ring, which is centered at $Q \approx \sqrt{2 \omega} \approx 2.7$ a.u. The ring is surrounded by two regions with a negative signal. Second, it should be noted that the scattering pattern is not symmetric. In that respect, the Compton DDSCS differs from the differential coherent scattering cross section [Eq. (1)], which is centrosymmetric according to Friedel's law. Finally, both the positive-valued ring and the negative-valued regions around it seem to oscillate with time.

In the following, we explain how those features mentioned above arise from characteristic features of the electron momentum-space density. For this purpose, let us consider the following decomposition of the momentum-space density in terms of the Hartree-Fock ground state $\rho_{0}(\mathbf{p})$, the density of the excited electron $\rho_{\text {elec }}(\mathbf{p}, t)$, and the density of the ionic hole $\rho_{\text {hole }}(\mathbf{p}, t)$ :

$$
\begin{aligned}
\rho(\mathbf{p}, t)= & \rho_{0}(\mathbf{p})+\rho_{\text {elec }}(\mathbf{p}, t)-\rho_{\text {hole }}(\mathbf{p}, t) \\
& +2 \operatorname{Re}\left\{\alpha_{0}(t) \sum_{i, a} \alpha_{i}^{a}(t) \varphi_{i}(\mathbf{p})^{*} \varphi_{a}(\mathbf{p})\right\} .
\end{aligned}
$$

Besides the densities of the excited electron and the ionic hole, there also appears an interference term between the excited electron and the hole. We show the different contributions in Fig. 3. Additionally, we show the sum of all three contributions in Figs. 3(m)-3(p). This corresponds to the changes of the electron momentum-space density with respect to the HartreeFock ground state. In all panels, we multiplied the respective contribution to the momentum-space density by the Jacobian (a)

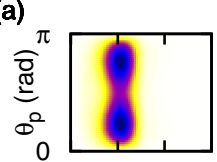

(e)

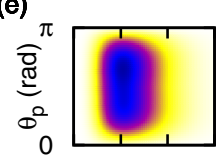

(i)

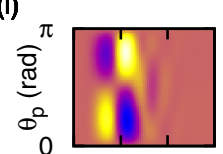

(m)

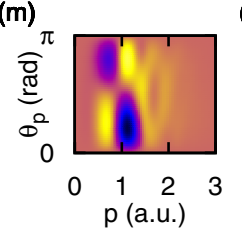

(b)

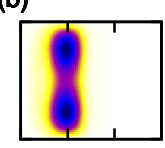

(f)

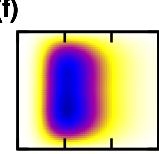

(j)

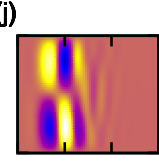

(n)

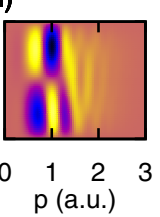

(c)

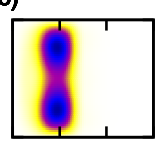

(g)

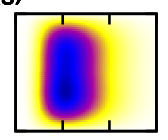

(k)

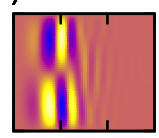

(o)

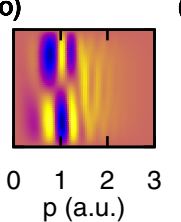

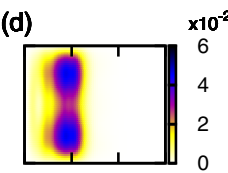

(h)

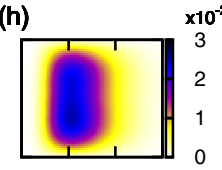

(I)

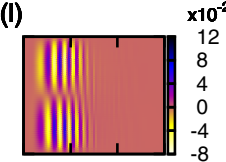

(p)

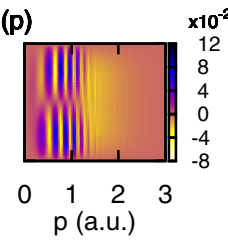

FIG. 3. Contributions to the electron momentum-space density. Panels (a)-(d) show the momentum-space density of the excited electron $\left[p^{2} \sin \left(\theta_{p}\right) \rho_{\text {elec }}(\mathbf{p}, t)\right]$ at delay times (a) $\tau_{\text {delay }}=6.9$ a.u., (b) 10.9 a.u., (c) 13.9 a.u., and (d) 36.9 a.u. (d). In panels (e)-(h) we display the momentum-space density of the hole $\left[p^{2} \sin \left(\theta_{p}\right) \rho_{\text {hole }}(\mathbf{p}, t)\right]$ at the same delay times. Panels (i)-(1) contain the contribution of the interference term in Eq. (23) [multiplied by the Jacobian $p^{2} \sin \left(\theta_{p}\right)$ ], and panels (m)-(p) show the sum of all three contributions. Note that panels in the same column all refer to the same delay time. $p^{2} \sin \left(\theta_{p}\right)$. First of all, it can be seen from Figs. 3(a)-3(d) that the momentum-space density of the excited electron is restricted to momenta with a magnitude smaller than 1.4 a.u. On the other hand, the momentum-space density of the ionic hole is more smeared out and extends up to momenta with a magnitude larger then 2 a.u., as can be seen in Figs. 3(e)-3(h). This behavior is a consequence of the fact that bound orbitals are strongly localized in real space. Hence, their Fourier transform will be more smeared out than that of an orbital that is not occupied in the Hartree-Fock ground state. To understand the ring structure of the DDSCS, note that the function $W_{1}$ in Eq. (14) is positive for all real arguments. Hence, there is a close connection between the signs of the DDSCS and the instantaneous momentum density. If the sign of the DDSCS is positive (negative), this means that the instantaneous momentum density is mainly positive (negative) at the momentum-space points that contribute to it. Moreover, one can see that the function $W_{1}$ in Eq. (14) can take its maximum value even for $P=0$, given that $Q \approx \sqrt{2 \omega}$ is fulfilled. This magnitude of $\mathbf{Q}$ corresponds approximately to the mean radius of the positive-valued ring. The more $Q$ deviates from this value, the larger $P$ needs to be, in order for $W_{1}$ to take on its maximum value. Hence, the regions of momentum space that are occupied by the excited electron can only be probed by momentum transfers with a magnitude in a small interval around $Q \approx \sqrt{2 \omega}$. On the other hand, the momentum-space density of the hole can also be probed by other momentum transfers $\mathbf{Q}$ because it extends into other regions of momentum space. Of course, one needs to consider the particle-hole interference term in Eq. (23), as well. As can be seen in Fig. 3, it can have a large influence on the total momentum-space density. However, the argument made above can still be applied. The interference term can only have a nonzero value if both the orbitals of the excited electron and the hole are nonzero at the considered momentum-space point. Additionally, one can also see the larger delocalization of the hole contribution in the total momentum-space density in Figs. 3(m)-3(p). Therefore, we conclude that the ringlike structure of the DDSCS reflects the fact that the instantaneous momentum density of the excited electron is localized at small momenta, whereas the instantaneous momentum density of the ionic hole also extends to momenta with a larger magnitude.

The asymmetry of the DDSCS directly results from the asymmetry of the electron momentum distribution. When comparing Fig. 2 with Figs. 3(m)-3(p), we can see that, whenever the upper half volume of momentum space $\left(\theta_{p} \leqslant \pi / 2\right)$ is occupied mainly by the excited electron $\left[\rho(\mathbf{p}, t)-\rho_{0}(\mathbf{p})>0\right]$, the positive-valued ring is more pronounced for momentum transfers with $Q_{z}>0$, and vice versa. The same relationship holds for the outer negative-valued ring. As we can see, it is more pronounced for negative values of $Q_{z}$ when the momentum-space density is mainly negative in the lower half volume, and vice versa. From a physical point of view, it might be surprising that the electron momentum-space density is that asymmetric in the first place. We see that the hole density displays only a slight asymmetry. This asymmetry stems from the overlap between the $3 s$ and $3 p$ orbitals, which have opposite parity. However, the asymmetry is necessarily small, because of the small population of the $3 s$ hole state. As a consequence, this slight asymmetry cannot explain 
the pronounced asymmetry visible in Figs. 3(m)-3(p). The momentum-space density of the excited electron does not display any visible asymmetry. Theoretically speaking, it is possible that asymmetry arises when an electron from the same occupied orbital can be excited into a number of states with different parity due to multiphoton effects. However, these excited states have different energies and are separated in momentum space. Hence, they have no notable overlap, and therefore they cannot contribute to an antisymmetric contribution to the momentum-space density. The pronounced asymmetry of the instantaneous momentum density is caused by the interference term between the excited electron and the ionic hole. For a one-photon process, the excited electron always has a parity opposite to that of the remaining hole, due to dipole selection rules. Note that the product of two orbitals with the same parity is necessarily symmetric. Hence, we see that the instantaneous momentum density is not symmetric, even if all excited electrons (holes) would behave the same under inversion.

Lastly, let us consider the temporal oscillations visible in Fig. 2. As we can see, both the momentum-space density of the excited electron and of the ionic hole change little with time. The dynamics of the momentum-space density, which correspond to the dynamics visible in the DDSCS, are largely driven by the interference term between the excited electron and the ionic hole. As we can see, the interference term displays oscillations with respect to $p$. Their frequency grows as time increases. This can be explained with the time evolution of the interference term, which is given by $\cos \left(\varepsilon_{a}-\varepsilon_{i}\right) t$. For free electrons, with momentum $\mathbf{p}, \varepsilon_{a}$ is given by $p^{2} / 2$. Thus, the interference term between the ionic hole and the excited electron will be proportional to $\cos \left(\frac{p^{2}}{2} t+\tilde{\phi}\right)$. This causes oscillations with respect to $p$ whose frequency increases with time. Additionally, one can see oscillations with respect to $\theta_{p}$. Those can be attributed to the parity of the hole orbital. The XUV pulse creates mainly holes in the $3 p$ orbitals. These change their sign under inversion, which gives rise to the observable oscillations.

The interference term has interesting consequences for the dynamics that can be seen in the DDSCS. For small times, one expects that the interference term gives a large contribution. This is especially true when the ground state is only weakly depopulated because then $\left|\alpha_{0}(t)\right| \gg\left|\alpha_{i}^{a}(t)\right|$ holds for all indices $(i, a)$. With increasing time, its contribution is expected to become small because the integral in Eq. (14) has to be performed for a more and more oscillatory function. This effect can be seen very clearly in Fig. 4. There, we display cuts through the DDSCS with the $x$ component of the momentum transfer fixed to a value of 0.07 a.u. Figure 4(a) shows cuts for the same delay times as in Fig. 2. We can see complex dynamics. The maxima oscillate while changing magnitude. At $\tau_{\text {delay }}=10.9$ a.u., a broadening of the profile can be seen, and a second structure appears next to the peaks. In Fig. 4(b), we plot the same quantity for delay times 45.9 a.u., 49.9 a.u., and 66.9 a.u. One can see that shape, position, and height of the peaks change only slightly. This indicates that, for short times, the dynamics in the DDSCS are largely driven by the interference term between the ionic hole and the excited electron. For larger times, its contribution decreases, and the DDSCS becomes almost stationary. A

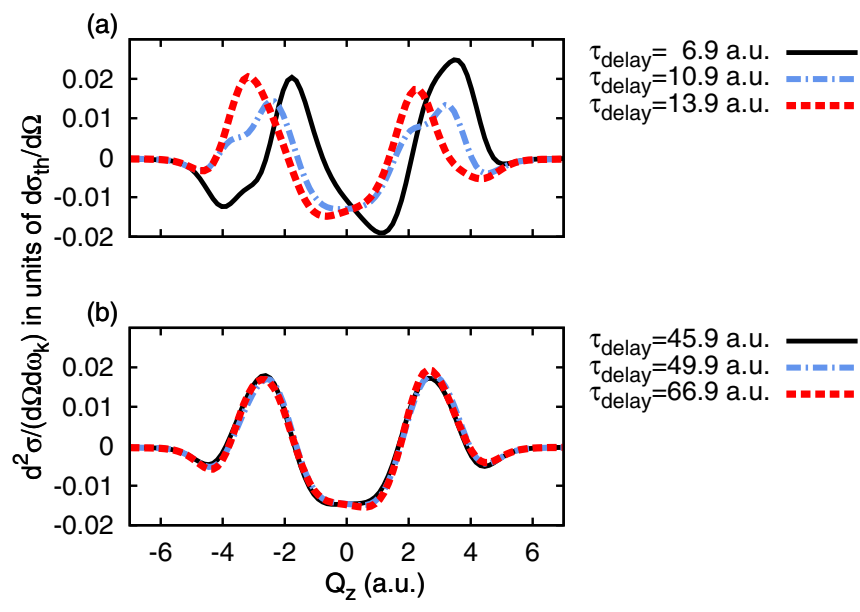

FIG. 4. Cuts through the DDSCS for $Q_{x}=0.07$ a.u. and $Q_{y}=0$ a.u. Energy transfer $\omega$ and pulse duration $T_{R}$ are the same as in Fig. 2. The delay times displayed are (a) $\tau_{\text {delay }}=6.9$ a.u, 10.9 a.u., 13.9 a.u., and (b) 45.9 a.u., 49.9 a.u., 66.9 a.u. The DDSCS is given in units of $d \sigma_{\mathrm{th}} / d \Omega$.

similar effect would be expected when using a simple Fourier picture. In that case, the overlap between the excited electron and the parent ion naturally becomes smaller when the electron moves away from the nucleus. However, the corresponding signal has a different physical origin, in both cases. In the case of coherent scattering, the decreasing overlap reflects how the excited electron moves away from the parent ion. In Compton scattering, the corresponding signal reflects the dispersion of the wave packet describing the ionized electron. Note that the contribution of the interference term will not vanish completely. In general, the oscillations will not cancel each other completely, if the bandwidth of the pulse is sufficiently small. Moreover, the excited electron can also occupy Rydberg states. Those are stationary states with a discrete eigenenergy. Thus, the argument made above does not apply and we cannot expect that the interference term between such a state and a hole state displays similar oscillations.

In the example considered here, we found three effects which characterize the appearance and the dynamics of the DDSCS. All of them are related to different aspects of the electron instantaneous momentum density. This illustrates the potential of time-resolved Compton scattering. Moreover, our results show how time-resolved scattering patterns can change when considering a more complex system. If one considers only coherent superpositions of one-electron states, this is equivalent to considering only an excited electron but neglecting the remaining electrons in the parent ion. However, interference with the residual ion notably influences the dynamics of the system and also the scattering patterns. Only for larger times can the dynamics of the bound electrons be treated separately from those of the ionized electrons.

In Fig. 5, we show the real-space density $\left[r^{2} \sin \left(\theta_{r}\right) \rho(\mathbf{x})\right]$ with the density of the Hartree-Fock ground state subtracted. One can clearly see how a particle-hole wave packet is formed and how the particle propagates away from the residual ion while dispersing. In Fig. 5(a), one sees that the density of the excited electron is asymmetric, while it is still in the vicinity of 
(a)

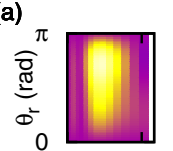

(b)

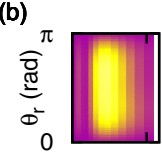

(c)

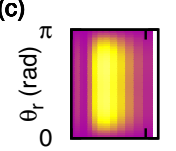

(d)

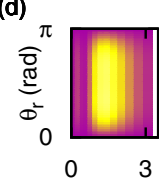

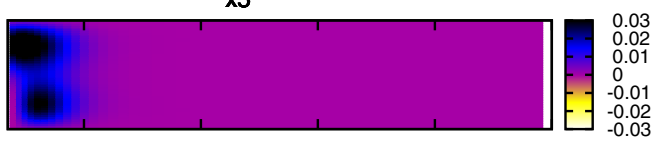

$\mathbf{x} 5$

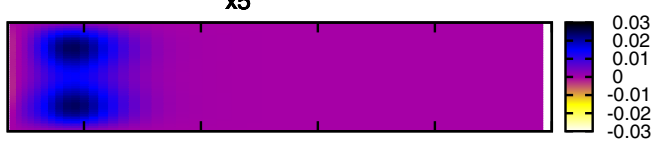

$\mathbf{x} 5$

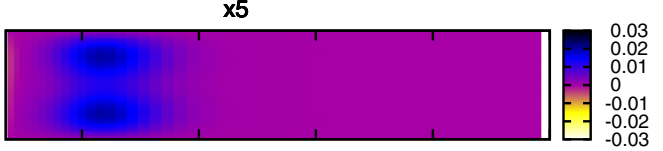

$\mathrm{x} 5$

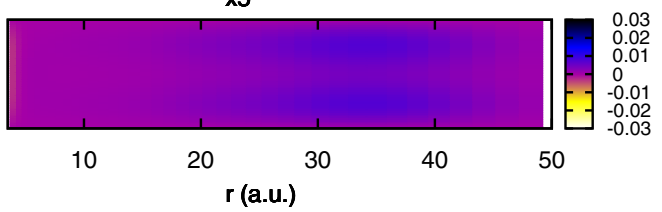

FIG. 5. Real-space density $\left[r^{2} \sin \left(\theta_{r}\right) \rho(\mathbf{x})\right]$ at delay times (a) $\tau_{\text {delay }}=6.9$ a.u., (b) 10.9 a.u., (c) 13.9 a.u., and (d) 36.9 a.u. The result for the Hartree-Fock ground state has been subtracted.

the parent ion. For larger times, the overlap between the particle and the hole becomes small and the density of the excited electron becomes symmetric. Similarly, one sees dynamics in the negative part of the real-space density for short times after the interaction with the XUV pulse [Figs. 5(a)-5(b)]. For larger times the changes become small.

\section{B. Results for two pump pulses}

As can be seen in Fig. 1, there is only a small degree of coherence between the $3 s$ and the $3 p_{0}$ hole, after excitation of the argon by one XUV pulse. Moreover, the $3 s$ hole remains almost unpopulated. To increase the degree of coherence, we use a second pulse that is in resonance with the $3 s-3 p_{0}$ transition $\left(\omega_{2}=13.47 \mathrm{eV}\right)$ of the argon ion. The peak electricfield strength is $84 \mathrm{GV} / \mathrm{m}$, and the pulse has a duration of 200 as. The electric field of the pulse sequence is plotted in Fig. 6(b). The duration of the second pulse is chosen in such a way that population is transferred from the $3 p_{0}$ hole to the $3 s$ hole until both states have a similar population. In Fig. 6(a) we plot the population of the Hartree-Fock ground state and some valence-shell holes. Moreover, we plot the degree of coherence between the $3 s$ and $3 p_{0}$ holes in Fig. 6(b). One sees that the degree of coherence is increased to $\approx 0.8$ after interaction with the both pulses. Also, the population of the $3 \mathrm{~s}$ hole is increased to 0.16 due to population transfer from the $3 p_{0}$ hole. However, one also sees that the population of the $3 p_{0}$ hole is increased to a value slightly higher than 0.2 instead of being reduced. Alongside with this, one sees a rapid depopulation of the Hartree-Fock ground state. Note that the mean photon energy of the pulse is lower than the ionization threshold of argon. Nevertheless, a certain amount of ionization can take place due to higher-energy photons within the bandwidth of the second pulse.

We also find a depopulation of the ground state when only the second pulse interacts with argon atoms in their
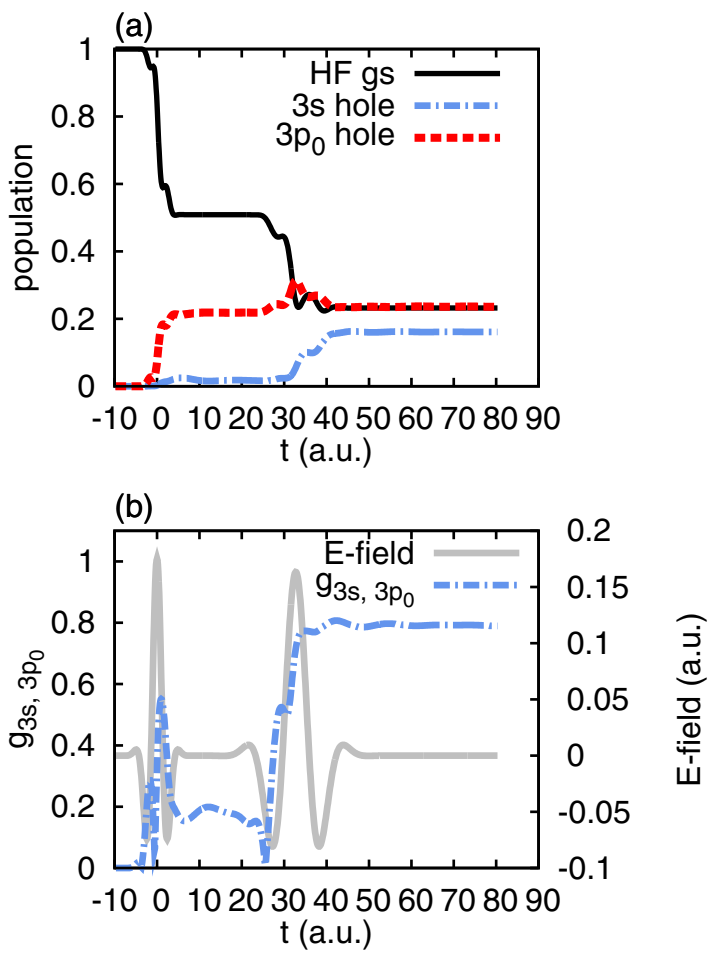

FIG. 6. Elements of the ionic density matrix for atomic Ar excited by a sequence of two XUV pulses. Panel (a) shows the populations of the Hartree-Fock ground state (solid black line), the $3 s$ hole (dotteddashed blue line), and the $3 p_{0}$ hole (dashed red line). In panel (b) we show the degree of coherence between the $3 s$ and $3 p_{0}$ hole (dotteddashed blue line) and the XUV electric field (solid gray line).

Hartree-Fock ground state. The same is true for longer pulse durations and even for a quasimonochromatic pulse. Therefore, the depopulation cannot be caused solely by the high-energy photons within the bandwidth of the second pulse. The additional excitations can be explained with the occupation of Rydberg states. Indeed, the photoabsorption spectrum of argon shows Rydberg lines at 11.9 and $14.1 \mathrm{eV}$ [48]. The second one of those is separated by only $0.6 \mathrm{eV}$ from the mean photon energy of the second XUV pulse. When varying the intensity of the second pulse, we find that the depopulation grows linearly for intensities smaller than $13 \mathrm{TW} / \mathrm{cm}^{2}$. For larger intensities, it grows more slowly due to saturation. This observation suggests that the additional depopulation of the ground state is not caused by multiphoton absorption. Thus, we conclude that the excitation of Rydberg states is responsible for the depopulation of the ground state.

In Fig. 7 we show the different contributions to the electron momentum-space density introduced in Eq. (23). As one can see in Figs. 7(a)-7(c), the instantaneous momentum density of the excited electron remains fairly stationary. It shows different bands due to the different kinds of excitations created by the two pump pulses. On the other hand, one can observe an upand-down motion in the instantaneous momentum density of the ionic hole [Figs. 7(d)-7(f)]. This wiggling motion appears because the pulses create an almost coherent superposition of two states with opposite parity. In the sum of all contributions [Figs. 7(j)-7(1)], we see that the overall appearance of the momentum-space density is determined mainly by $\rho_{\text {elec }}(\mathbf{p}, t)$ 
(a)

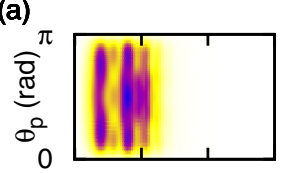

(d)

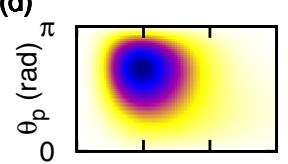

(g)

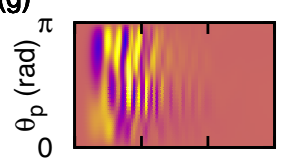

(j)

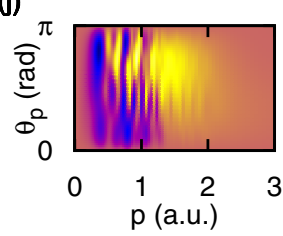

(b)

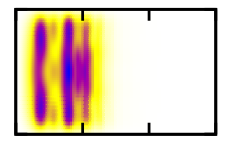

(e)

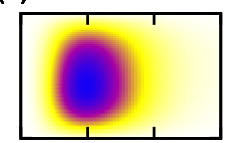

(h)

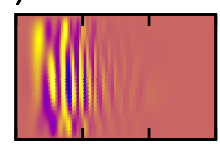

(k)

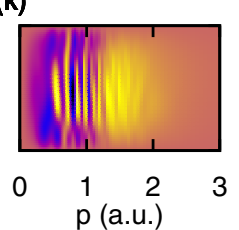

(c)

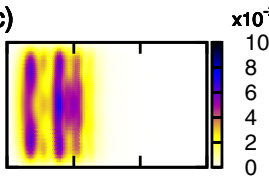

(f)

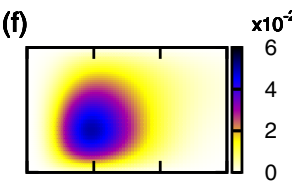

(i)

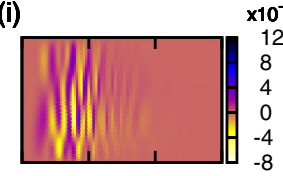

(I)

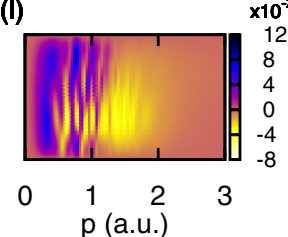

FIG. 7. Contributions to the electron momentum-space density. Panels (a)-(c) show the momentum-space density of the excited electron $\left[p^{2} \sin \left(\theta_{p}\right) \rho_{\text {elec }}(\mathbf{p}, t)\right]$ at delay times (a) $\tau_{\text {delay }}=51.2$ a.u., (b) 55.2 a.u., and (c) 58.2 a.u. In panels (d)-(f) we display the momentum-space density of the hole $\left[p^{2} \sin \left(\theta_{p}\right) \rho_{\text {hole }}(\mathbf{p}, t)\right]$ at the same delay times. Panels (g)-(i) contain the contribution of the interference term in Eq. (23) [multiplied by the Jacobian $p^{2} \sin \left(\theta_{p}\right)$ ], and panels (j)-(l) show the sum of all three contributions. Note that panels in the same column all refer to the same delay time.

and $\rho_{\text {hole }}(\mathbf{p}, t)$. In contrast to Sec. IV A, the interference term plays a less important role.

In Fig. 8, we show the DDSCS after interaction of the argon atom with both pump pulses. The scattering parameters are the same as before. One can clearly see how both the positive and the negative peaks oscillate up and down, thus reflecting the wiggling motion of the hole. The oscillations have a period of $\sim 13$ a.u. With our time resolution, this corresponds well to the expected period of $2 \pi /\left|\varepsilon_{3 s}-\varepsilon_{3 p_{0}}\right| \approx 12.7$ a.u. It should be stressed that the changes in the positive-valued ring are not caused by the dynamics of the excited electron. Instead, the changes appear because the momentum distributions of the excited electrons and the ionic holes are overlapping. In consequence, the ionic hole eclipses the momentum-space density of the excited electron in regions where the former is localized, as can be seen in Figs. 7(j)-7(1). This behavior is of particular interest if one tries to image the dynamics of the ionic hole. As we have seen, the pumping process with two XUV pulses induces fairly complicated dynamics with various kinds of excitations. However, the dynamics in the scattering patterns mainly reflects the wiggling motion of the hole.

\section{CONCLUSION}

We investigated the imaging of electron-hole wave-packet dynamics in argon atoms. Specifically, we considered the
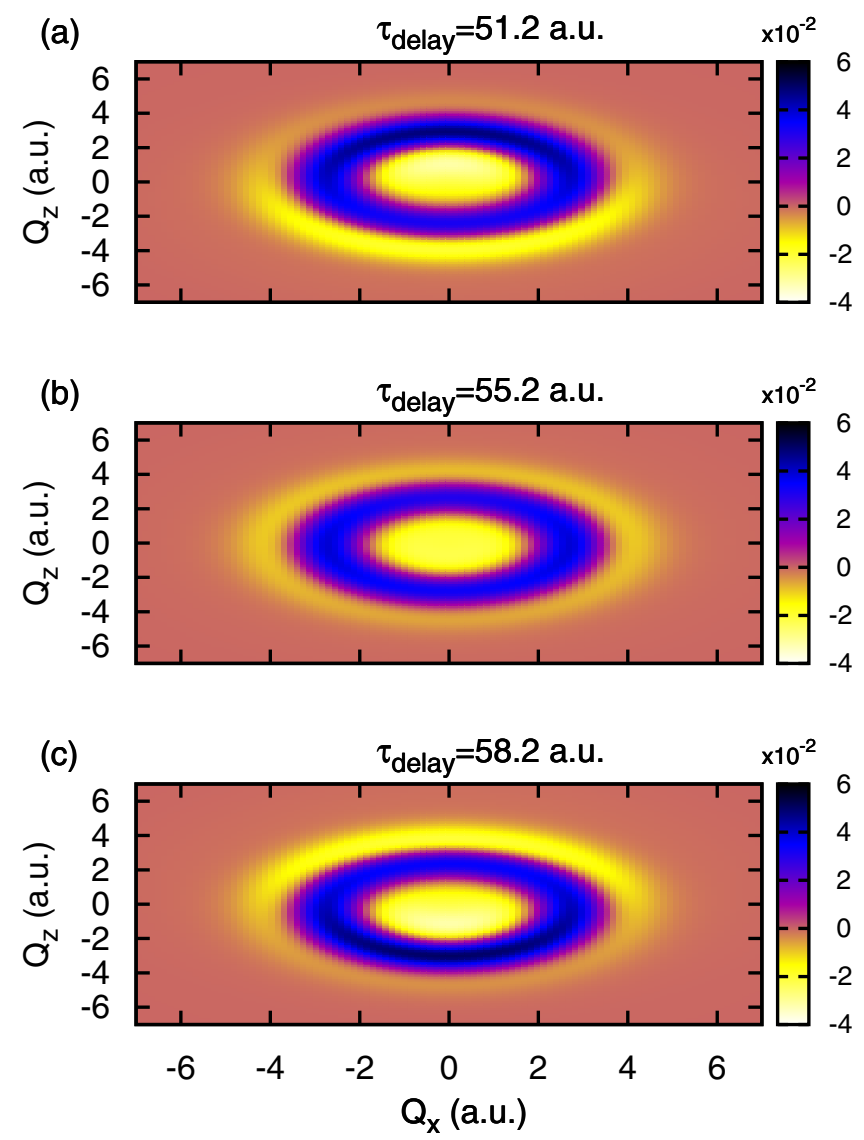

FIG. 8. DDSCS for an energy transfer $\omega=100 \mathrm{eV}$ and $T_{R}=23.5$ as at delay times (a) $\tau_{\text {delay }}=51.2$ a.u., (b) 55.2 a.u., and (c) 58.2 a.u. in units of $d \sigma_{\mathrm{th}} / d \Omega$. The $y$ component of the momentum-transfer vector is set to zero.

signal caused by inelastically scattered photons, which are inevitable in time-resolved x-ray scattering experiments. For this purpose, we developed a QED-based theory of timeresolved Compton scattering. The Compton-scattering cross section, in the impulse approximation, depends solely on the electron momentum distribution. Hence, time-resolved Compton scattering can be used to obtain momentum-space images of the sample to be probed. To illustrate this, we first considered an electronic wave packet created through ionization or Rydberg excitation of argon atoms by an XUV pulse. We showed how characteristic features of the instantaneous momentum density are reflected in the doubly differential scattering cross section. This illustrates the kind of information that can be gained from time-resolved Compton scattering. Interestingly, the dynamics of the ionized electron cannot be treated separately from the dynamics of the ionic hole. Instead, both the instantaneous momentum density and the DDSCS are strongly influenced by interference between the particle and the hole.

Additionally, we considered an almost coherent superposition of the $3 s$ and $3 p_{0}$ holes, generated by a sequence of two XUV pulses. Even though the dynamics of the excited electrons are fairly complex, the DDSCS clearly reflects the wiggling motion of the ionic hole. We expect that imaging the hole motion is also possible, when finite-energy resolution 
is granted. Only when there is no energy resolution, the differential scattering cross section becomes stationary. Thus, imaging the dynamics of the holes via time-resolved Compton scattering is no longer possible.

Our results for the time-dependent Compton-scattering cross sections rely on the assumption that the recoil electron is not correlated with the remaining ion. Hence, in our results for the differential scattering cross section, there could be deviations due to photons scattered with small energy transfer. However, those mainly contribute to scattering at small scattering angles, where contributions due to coherent scattering are dominant. Therefore, the conclusions we draw should still be valid.

We conclude that time-resolved Compton scattering can indeed be used for imaging of electron-hole wave-packet dynamics. Since it focuses on momentum space, it can yield complementary information to techniques that focus on the real-space density of the electrons.
[1] H. Ibach and H. Lüth, Solid-State Physics: An Introduction to Principles of Materials Science, 4th ed. (Springer, Berlin, Heidelberg, 2009).

[2] D. Griffiths, Introduction to Elementary Particles (Wiley-VCH, Weinheim, 2008).

[3] C. Kittel, Introduction to Solid State Physics, 8th ed. (Wiley, New York, 2005).

[4] S. Blundell, Magnetism in Condensed Matter (Oxford University Press, Oxford, 2003).

[5] C. Hammond, The Basics of Crystallography and Diffraction, 4th ed., IUCr Texts on Crystallography (Oxford University Press, Oxford, 2015).

[6] W. Schuelke, Electron Dynamics by Inelastic X-Ray Scattering (Oxford University Press, Oxford, 2007).

[7] H. N. Chapman, A. Barty, M. J. Bogan, S. Boutet, M. Frank, S. P. Hau-Riege, S. Marchesini, B. W. Woods, S. Bajt, W. H. Benner, R. A. London, E. Plönjes, M. Kuhlmann, R. Treusch, S. Düsterer, T. Tschentscher, J. R. Schneider, E. Spiller, T. Möller, C. Bostedt, M. Hoener, D. A. Shapiro, K. O. Hodgson, D. van der Spoel, F. Burmeister, M. Bergh, C. Caleman, G. Huldt, M. M. Seibert, F. R. N. C. Maia, R. W. Lee, A. Szöke, N. Timneanu, and J. Hajdu, Nat. Phys. 2, 839 (2006).

[8] B. W. J. McNeil and N. R. Thompson, Nat. Photon. 4, 814 (2010).

[9] M. J. J. Vrakking and T. Elsaesser, Nat. Photon. 6, 645 (2012).

[10] P. B. Corkum and F. Krausz, Nat. Phys. 3, 381 (2007).

[11] F. Krausz and M. Ivanov, Rev. Mod. Phys. 81, 163 (2009).

[12] S. Kumar, H.-S. Kang, and D.-E. Kim, Appl. Sci. 3, 251 (2013).

[13] E. L. Saldin, E. A. Schneidmiller, and M. V. Yurkov, Phys. Rev. Spec. Top.-Accel. Beams 9, 050702 (2006).

[14] E. Prat and S. Reiche, Phys. Rev. Lett. 114, 244801 (2015).

[15] A. A. Zholents, Phys. Rev. Spec. Top.-Accel. Beams 8, 040701 (2005).

[16] P. Emma, K. Bane, M. Cornacchia, Z. Huang, H. Schlarb, G. Stupakov, and D. Walz, Phys. Rev. Lett. 92, 074801 (2004).

[17] A. A. Zholents and W. M. Fawley, Phys. Rev. Lett. 92, 224801 (2004).

[18] T. Tanaka, Phys. Rev. Lett. 110, 084801 (2013).

[19] S. R. Leone, C. W. McCurdy, J. Burgdorfer, L. S. Cederbaum, Z. Chang, N. Dudovich, J. Feist, C. H. Greene, M. Ivanov, R. Kienberger, U. Keller, M. F. Kling, Z.-H. Loh, T. Pfeifer, A. N. Pfeiffer, R. Santra, K. Schafer, A. Stolow, U. Thumm, and M. J. J. Vrakking, Nat. Photon. 8, 162 (2014).

[20] M. Schultze, M. Fieß, N. Karpowicz, J. Gagnon, M. Korbman, M. Hofstetter, S. Neppl, A. L. Cavalieri, Y. Komninos, T. Mercouris, C. A. Nicolaides, R. Pazourek, S. Nagele, J. Feist, J. Burgdörfer, A. M. Azzeer, R. Ernstorfer, R. Kienberger,
U. Kleineberg, E. Goulielmakis, F. Krausz, and V. S. Yakovlev, Science 328, 1658 (2010).

[21] R. Pazourek, J. Feist, S. Nagele, and J. Burgdörfer, Phys. Rev. Lett. 108, 163001 (2012).

[22] C.-H. Zhang and U. Thumm, Phys. Rev. A 82, 043405 (2010).

[23] A. L. Cavalieri, N. Muller, T. Uphues, V. S. Yakovlev, A. Baltuska, B. Horvath, B. Schmidt, L. Blumel, R. Holzwarth, S. Hendel, M. Drescher, U. Kleineberg, P. M. Echenique, R. Kienberger, F. Krausz, and U. Heinzmann, Nature (London) 449, 1029 (2007).

[24] C. Lemell, B. Solleder, K. Tőkési, and J. Burgdörfer, Phys. Rev. A 79, 062901 (2009).

[25] E. Goulielmakis, Z.-H. Loh, A. Wirth, R. Santra, N. Rohringer, V. S. Yakovlev, S. Zherebtsov, T. Pfeifer, A. M. Azzeer, M. F. Kling, S. R. Leone, and F. Krausz, Nature (London) 466, 739 (2010).

[26] S. Haessler, J. Caillat, and P. Salières, J. Phys. B: At., Mol. Opt. Phys. 44, 203001 (2011).

[27] O. Smirnova, Y. Mairesse, S. Patchkovskii, N. Dudovich, D. Villeneuve, P. Corkum, and M. Y. Ivanov, Nature (London) 460, 972 (2009).

[28] A. D. Dutoi, M. Wormit, and L. S. Cederbaum, J. Chem. Phys. 134, 024303 (2011).

[29] S. Pabst, L. Greenman, P. J. Ho, D. A. Mazziotti, and R. Santra, Phys. Rev. Lett. 106, 053003 (2011).

[30] G. Dixit, O. Vendrell, and R. Santra, Proc. Natl. Acad. Sci. USA 109, 11636 (2012).

[31] H. J. Suominen and A. Kirrander, Phys. Rev. Lett. 112, 043002 (2014).

[32] R. Santra, G. Dixit, and J. M. Slowik, Phys. Rev. Lett. 113, 189301 (2014).

[33] H. J. Suominen and A. Kirrander, Phys. Rev. Lett. 113, 189302 (2014).

[34] H.-C. Shao and A. F. Starace, Phys. Rev. A 88, 062711 (2013).

[35] H.-C. Shao and A. F. Starace, Phys. Rev. A 87, 050701 (2013).

[36] H.-C. Shao and A. F. Starace, Phys. Rev. A 90, 032710 (2014).

[37] G. Dixit, J. M. Slowik, and R. Santra, Phys. Rev. Lett. 110, 137403 (2013).

[38] A. F. Kemper, M. Sentef, B. Moritz, C. C. Kao, Z. X. Shen, J. K. Freericks, and T. P. Devereaux, Phys. Rev. B 87, 235139 (2013).

[39] G. L. Yudin, D. I. Bondar, S. Patchkovskii, P. B. Corkum, and A. D. Bandrauk, J. Phys. B: At., Mol. Opt. Phys. 42, 205601 (2009).

[40] M. J. Cooper, Rep. Prog. Phys. 48, 415 (1985).

[41] G. P. Das, K. V. Bhagwat, and V. C. Sahni, Phys. Rev. A 36, 2984 (1987).

[42] P. Bergstrom and R. Pratt, Radiat. Phys. Chem. 50, 3 (1997). 
[43] R. Pratt, L. LaJohn, T. Suric, B. Chatterjee, and S. Roy, Nucl. Instrum. Methods Phys. Res., Sect. B 261, 175 (2007).

[44] L. Greenman, P. J. Ho, S. Pabst, E. Kamarchik, D. A. Mazziotti, and R. Santra, Phys. Rev. A 82, 023406 (2010).

[45] N. Rohringer, A. Gordon, and R. Santra, Phys. Rev. A 74, 043420 (2006).

[46] S. Pabst and R. Santra, Phys. Rev. Lett. 111, 233005 (2013).

[47] T. Mazza, A. Karamatskou, M. Ilchen, S. Bakhtiarzadeh, A. J. Rafipoor, P. O’Keeffe, T. J. Kelly, N. Walsh, J. T. Costello, M. Meyer, and R. Santra, Nat. Commun. 6, 6799 (2014).

[48] D. Krebs, S. Pabst, and R. Santra, Am. J. Phys. 82, 113 (2014).

[49] S. Pabst, A. Sytcheva, A. Moulet, A. Wirth, E. Goulielmakis, and R. Santra, Phys. Rev. A 86, 063411 (2012).

[50] S. Pabst, Eur. Phys. J. Spec. Top. 221, 1 (2013).

[51] F. Schwabl, Advanced Quantum Mechanics (Springer, Berlin, Heidelberg, 2005).

[52] A. Fetter and J. Walecka, Quantum Theory of Many-Particle Systems (Dover Publications, Mineola, 2003).

[53] N. H. March, W. H. Young, and S. Sampanthar, The ManyBody Problem in Quantum Mechanics (Dover Publications, Mineola, 1967).

[54] D. Craig and T. Thirunamachandran, Molecular Quantum Electrodynamics (Dover Publications, Mineola, 1998)

[55] R. Loudon, The Quantum Theory of Light, 3rd ed. (Oxford University Press, Oxford, 2000).

[56] R. Santra, J. Phys. B: At., Mol. Opt. Phys. 42, 023001 (2009).

[57] J. M. Slowik and R. Santra, J. Phys. B: At., Mol. Opt. Phys. 46, 164016 (2013).

[58] G. Dixit, J. M. Slowik, and R. Santra, Phys. Rev. A 89, 043409 (2014).

[59] I. G. Kaplan, B. Barbiellini, and A. Bansil, Phys. Rev. B 68, 235104 (2003).

[60] P. Eisenberger and P. M. Platzman, Phys. Rev. A 2, 415 (1970).

[61] G. Dixit and R. Santra, J. Chem. Phys. 138, 134311 (2013).

[62] L. Strüder, S. Epp, D. Rolles, R. Hartmann, P. Holl, G. Lutz, H. Soltau, R. Eckart, C. Reich, K. Heinzinger, C. Thamm,
A. Rudenko, F. Krasniqi, K.-U. Kühnel, C. Bauer, C.-D. Schröter, R. Moshammer, S. Techert, D. Miessner, M. Porro, O. Hälker, N. Meidinger, N. Kimmel, R. Andritschke, F. Schopper, G. Weidenspointner, A. Ziegler, D. Pietschner, S. Herrmann, U. Pietsch, A. Walenta, W. Leitenberger, C. Bostedt, T. Möller, D. Rupp, M. Adolph, H. Graafsma, H. Hirsemann, K. Gärtner, R. Richter, L. Foucar, R. L. Shoeman, I. Schlichting, and J. Ullrich, Nucl. Instrum. Methods Phys. Res., Sect. A 614, 483 (2010).

[63] H. T. Philipp, M. Hromalik, M. Tate, L. Koerner, and S. M. Gruner, Nucl. Instrum. Methods Phys. Res., Sect. A 649, 67 (2011).

[64] S. Boutet and G. J Williams, New J. Phys. 12, 035024 (2010).

[65] I. Waller and D. R. Hartree, Proc. R. Soc. London, Ser. A 124, 119 (1929).

[66] J. M. Slowik, S.-K. Son, G. Dixit, Z. Jurek, and R. Santra, New J. Phys. 16, 073042 (2014).

[67] Y.-J. Chen, S. Pabst, A. Karamatskou, and R. Santra, Phys. Rev. A 91, 032503 (2015).

[68] J. Breidbach and L. S. Cederbaum, J. Chem. Phys. 118, 3983 (2003).

[69] B. Podolsky and L. Pauling, Phys. Rev. 34, 109 (1929).

[70] H.-C. Shao and A. F. Starace, Phys. Rev. Lett. 105, 263201 (2010).

[71] D. Popova-Gorelova and R. Santra, Phys. Rev. B 91, 184303 (2015).

[72] D. Popova-Gorelova and R. Santra, Phys. Rev. B 92, 184304 (2015).

[73] A. A. Kramida, Y. Ralchenko, Y. J. Reader, and NIST ASD Team, NIST Atomic Spectra Database, Ver. 5.3 (online), Available at http://physics.nist.gov/asd (August 17, 2016), National Institute of Standards and Technology, Gaithersburg, Maryland, USA (2015).

[74] R. E. Goetz, A. Karamatskou, R. Santra, and C. P. Koch, Phys. Rev. A 93, 013413 (2016).

[75] R. E. Goetz, M. Merkel, A. Karamatskou, R. Santra, and C. P. Koch, Phys. Rev. A 94, 023420 (2016). 